\title{
Insights into the Role of Erysipelotrichaceae in the Human Host
}

\author{
Nadeem O. Kaakoush* \\ School of Biotechnology and Biomolecular Sciences, Faculty of Science, University of New South Wales, Sydney, NSW, \\ Australia
}

Keywords: Erysipelotrichaceae, gastrointestinal, microbiota, metabolic disorders, inflammatory bowel diseases, colorectal cancer

Understanding the human gut microbiota has garnered interest from researchers and clinicians to pharmaceutical companies looking at novel mechanisms to manipulate the microbiota for the benefit of the host. Studies on the gut microbiota can be loosely characterized into three areas that include investigating the microbiota's role in the physiology of the healthy gut, in the establishment of gastrointestinal disease, and in extra-intestinal manifestations. With deep sequencing technologies now in routine use in the research environment, novel members of the gut microbiota are coming to light, and our understanding of this complex ecosystem and its relationship to the host is slowly improving.

\section{OPEN ACCESS}

Edited by:

D. Scott Merrell,

Uniformed Services University of the Health Sciences, USA

Reviewed by:

Valerio lebba

'Sapienza' University of Rome, Italy Ryan Chandler Johnson, Henry M. Jackson Foundation for the Advancement of

Military Medicine, USA

Traci Testerman,

University of South Carolina, USA

*Correspondence:

Nadeem O. Kaakoush

n.kaakoush@unsw.edu.au

Received: 14 September 2015 Accepted: 09 November 2015 Published: 20 November 2015

Citation:

Kaakoush NO (2015) Insights into the Role of Erysipelotrichaceae in the Human Host.

Front. Cell. Infect. Microbiol. 5:84. doi: 10.3389/fcimb.2015.00084

\section{IMPORTANCE OF ERYSIPELOTRICHACEAE IN HUMANS}

Reports documenting a potential role for the bacterial family Erysipelotrichaceae, which belongs to the Firmicutes phylum, in host physiology and/or disease are on the rise. However, more often than not, these organisms are mentioned in passing, despite the fact that members of this bacterial family appear to be highly immunogenic and can potentially flourish post-treatment with broad spectrum antibiotics (Zhao et al., 2013; Palm et al., 2014; Dinh et al., 2015). For example, Palm and colleagues who developed a method to sort and sequence members of the intestinal microbiota based on coating with immunoglobulin A (IgA), termed IgA-SEQ, found one member of the Erysipelotrichaceae to be highly coated by IgA relative to other members of the gut microbiota (Palm et al., 2014). More recently, Ding and colleagues observed that the relative abundance of Erysipelotrichi positively correlated with tumor necrosis factor alpha (TNF) levels in a study investigating patients who had chronic HIV infection and were receiving suppressive antiretroviral therapy and HIV-uninfected controls (Dinh et al., 2015). Further, Zhao and colleagues found an increase in the levels of Erysipelotrichaceae incertae sedis in mice treated with high doses of gentamicin (Zhao et al., 2013).

\section{ERYSIPELOTRICHACEAE AND INFLAMMATION-RELATED GASTROINTESTINAL DISEASES}

The importance of Erysipelotrichaceae in inflammation-related disorders of the gastrointestinal tract is highlighted by the fact that they have been found to be enriched in colorectal cancer. For example, their abundance levels were found to be increased in the lumen of colorectal cancer patients as compared to healthy controls (Chen et al., 2012), and to be significantly higher in the tumor group of an animal model of 1, 2-dimethylhydrazine-induced colon cancer (Zhu et al., 2014). 
Changes in the levels of Erysipelotrichaceae in patients with inflammatory bowel diseases (IBD) or animal models of IBD have also been observed; however, the evidence does not appear to be consistent (Craven et al., 2012; Dey et al., 2013; Gevers et al., 2014; Labbé et al., 2014; Palm et al., 2014; Schaubeck et al., 2015). Craven and colleagues identified ileitis-associated shifts toward Erysipelotrichaceae in C57BL6 mice infected with Toxoplasma gondii or Giardia muris (Craven et al., 2012), while Schaubeck and colleagues observed significant increases in the abundance of Erysipelotrichaceae in mice that develop a TNF-driven Crohn's disease (CD)-like transmural inflammation (Schaubeck et al., 2015). These findings are of interest, given the association between Erysipelotrichaceae and TNF levels (Dinh et al., 2015).

In contrast, Dey and colleagues found that patients who experienced recurrence of CD had significantly lower levels of Erysipelotrichaceae (Dey et al., 2013), and similarly, Gevers and colleagues found a decreased abundance of Erysipelotrichales in patients within new-onset CD (Gevers et al., 2014). These findings are supported by a study by Labbe and colleagues who analyzed publicly available metagenomic datasets of IBD patients and controls for bile metabolizing genes, and found that the abundance of bile salt hydrolase genes originating from Firmicutes taxa including Erysipelotrichaceae was significantly reduced in IBD as compared to healthy controls (Labbé et al., 2014).

These results would suggest inter-host variation, with inflammatory mouse models having higher levels of Erysipelotrichaceae, and patients with IBD having lower levels. Two possible explanations for this include the inherent differences in the gut microbiota of mice and humans and/or differences in their innate immune responses upon sensing bacterial ligands (Zschaler et al., 2014; Nguyen et al., 2015). However, Palm and colleagues did not observe a significant difference in the abundance levels of a specific immunogenic Erysipelotrichaceae species between IBD patients and controls (Palm et al., 2014). Nonetheless, when the authors infected germ-free mice with a consortium of bacteria that were classified as highly coated by IgA (containing Erysipelotrichaceae spp.), these mice developed a more severe colitis upon treatment with dextran sodium sulfate than mice colonized by an IgAnegative consortium of bacteria (Palm et al., 2014). Thus, the inconsistencies in the results related to IBD could potentially be attributed to a lack of proper characterization of taxa belonging to the Erysipelotrichaceae family. For example, species within Erysipelotrichaceae may have diverse immunogenicity profiles or respond differently to inflammation within the gut.

\section{ERYSIPELOTRICHACEAE AND METABOLIC DISORDERS}

Perhaps the strongest evidence for a role for Erysipelotrichaceae in human disease comes from studies related to metabolic disorders. Early work on this topic showed a bloom of species belonging to Erysipelotrichaceae (classified as Mollicutes at the time of that study) in diet-induced obese animals (Turnbaugh et al., 2008). In addition, higher levels of Erysipelotrichaceae in obese individuals (Zhang et al., 2009), and a drop of approximately 2.5 -fold in the abundance of Erysipelotrichaceae taxa in a hamster model of hypercholesterolemia treated with an extract to improve cholesterol homeostasis (Martínez et al., 2009) have been observed. Subsequent studies have confirmed the association between this bacterial family and lipidemic profiles within the host. For example, Zhang and colleagues identified four different lineages within Erysipelotrichaceae to respond differently to diet or host health phenotypes (Zhang et al., 2010), while Fleissner and colleagues observed an increase of Erysipelotrichaceae in mice on high-fat or western diet (Fleissner et al., 2010). Further, Spencer and colleagues showed that the abundance of Erysipelotrichi were positively associated with changes in liver fat in female subjects who were placed on diets in which choline levels were manipulated (Spencer et al., 2011).

More recently, in an important study examining the microbiota of hamsters whose cholesterol metabolism was modulated using dietary plant sterol esters, Martinez and colleagues provided strong evidence for a correlation between the levels of Erysipelotrichaceae and host cholesterol metabolites (Martínez et al., 2013). In addition to this, Etxeberria and colleagues observed that supplementation of the flavonol quercetin inhibited the growth of Erysipelotrichaceae (Etxeberria et al., 2015), which is particularly interesting given that flavonoids have been suggested to assist in weight loss (Hurt and Wilson, 2012).

\section{NUTRITION STUDIES PROVIDE FURTHER INSIGHTS INTO THE ROLE OF ERYSIPELOTRICHACEAE IN THE GUT}

Nutrition studies further support the influence of dietary fat on the abundance of Erysipelotrichaceae. Harris and colleagues identified an accumulation of a specific taxon within Erysipelotrichaceae in mice with liver injury associated with parenteral nutrition, and observed a marked reduction in liver injury also corresponding to a decrease in the abundance of the same Erysipelotrichaceae taxon following antibiotic treatment (Harris et al., 2014). The authors determined that the fluctuations in abundance of Erysipelotrichaceae corresponded to the soy oil based-lipid emulsion from the parenteral nutrition solution (Harris et al., 2014).

In support of these findings, our group has recently observed a decrease in the abundance of Erysipelotrichaceae in CD patients undergoing low-fat ( $13.5 \mathrm{~g} / 1000 \mathrm{Kcal}$; Nahidi et al., 2013) exclusive enteral nutrition (EEN) therapy (Kaakoush et al., 2015). This is of interest when taken together with the findings of $\mathrm{Li}$ and colleagues who showed that EEN therapy was associated with a significant decrease in visceral fat area in patients with $C D$ (Li et al., 2014). Indeed, Gassull and colleagues have concluded that the type of dietary fat may be important for the efficacy of enteral nutrition in CD (Gassull et al., 2002). Given this, it is no surprise that IBD patients often exhibit differences in their levels of cholesterol as compared to healthy subjects (Agouridis et al., 2011), and infliximab maintenance therapy targeting TNF in $\mathrm{CD}$ patients, leads to an increase in cholesterol levels and a 


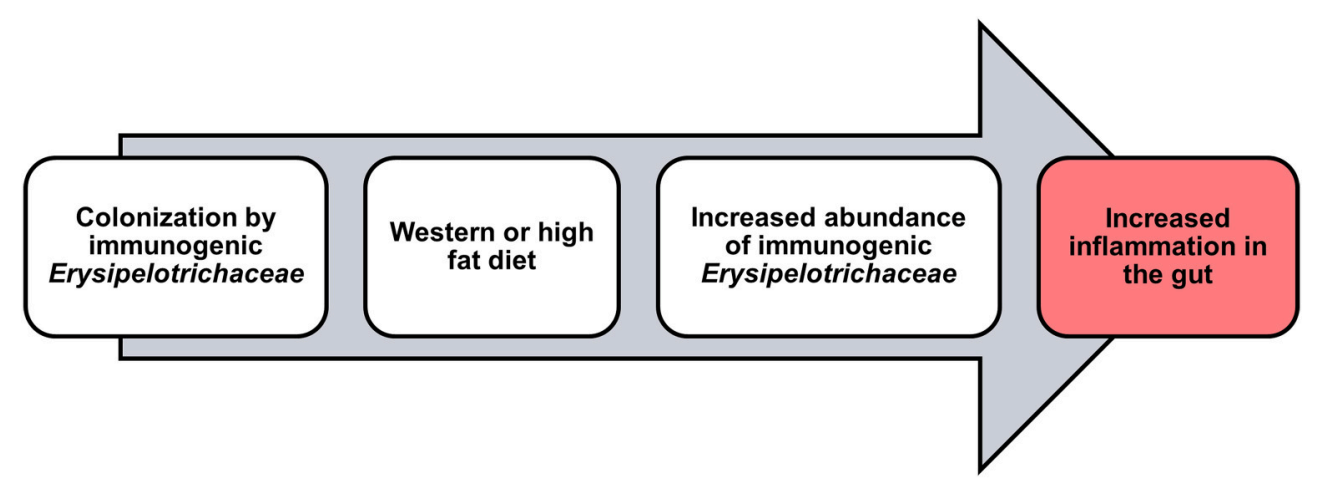

FIGURE 1 | Potential role for Erysipelotrichaceae in inflammation within the host.

significant increase in abdominal fat tissue (Parmentier-Decrucq et al., 2009). With all the above evidence in mind, the fluctuations in Erysipelotrichaceae levels in IBD patients are likely to be associated with patient lipid or cholesterol phenotypes and/or patient diet, but may still have a significant impact on disease presentation and/or activity (Figure 1).

\section{CONCLUSIONS}

Taken together, there is strong evidence supporting an association between Erysipelotrichaceae and host lipid metabolism, warranting additional investigation into the metabolic profiles of these organisms, and the influence they have on the host. Partial loss of fatty acid biosynthesis has been reported in the genomes of some members of Erysipelotrichaceae (e.g., Erysipelothrix rhusiopathiae; Kwok et al., 2014); thus, it would be of interest to determine if these metabolic characteristics have any impact on their role within the host. Further, the finding that specific taxa within Erysipelotrichaceae

\section{REFERENCES}

Agouridis, A. P., Elisaf, M., and Milionis, H. J. (2011). An overview of lipid abnormalities in patients with inflammatory bowel disease. Ann. Gastroenterol. 24, 181-187.

Chen, W., Liu, F., Ling, Z., Tong, X., and Xiang, C. (2012). Human intestinal lumen and mucosa-associated microbiota in patients with colorectal cancer. PLOS ONE 7:e39743. doi: 10.1371/journal.pone.0039743

Craven, M., Egan, C. E., Dowd, S. E., McDonough, S. P., Dogan, B., Denkers, E. Y., et al. (2012). Inflammation drives dysbiosis and bacterial invasion in murine models of ileal Crohn's disease. PLoS ONE 7:e41594. doi: 10.1371/journal.pone.0041594

Dey, N., Soergel, D. A., Repo, S., and Brenner, S. E. (2013). Association of gut microbiota with post-operative clinical course in Crohn's disease. BMC Gastroenterol. 13:131. doi: 10.1186/1471-230X-13-131

Dinh, D. M., Volpe, G. E., Duffalo, C., Bhalchandra, S., Tai, A. K., Kane, A. V., et al. (2015). Intestinal microbiota, microbial translocation, and systemic inflammation in chronic HIV infection. J. Infect. Dis. 211, 19-27. doi: 10.1093/infdis/jiu409

Etxeberria, U., Arias, N., Boqué, N., Macarulla, M. T., Portillo, M. P., Martínez, J. A., et al. (2015). Reshaping faecal gut microbiota composition by the intake may be correlated to inflammation (Dinh et al., 2015), while others are highly immunogenic (Palm et al., 2014) adds weight to the importance of properly characterizing this bacterial family, and may eventually provide promising microbial targets to combat metabolic disorders. It is worth noting, however, that current evidence associating this bacterial family with disease is correlative, and studies examining the direct impact that changes in the abundance of Erysipelotrichaceae have on the host are required.

\section{AUTHOR CONTRIBUTIONS}

NK conceived the idea and wrote the manuscript.

\section{ACKNOWLEDGMENTS}

NK would like to acknowledge support from the University of New South Wales.

of trans-resveratrol and quercetin in high-fat sucrose diet-fed rats. J. Nutr. Biochem. 26, 651-660. doi: 10.1016/j.jnutbio.2015.01.002

Fleissner, C. K., Huebel, N., Abd El-Bary, M. M., Loh, G., Klaus, S., and Blaut, M. (2010). Absence of intestinal microbiota does not protect mice from dietinduced obesity. Br. J. Nutr. 104, 919-929. doi: 10.1017/S0007114510001303

Gassull, M. A., Fernández-Bañares, F., Cabré, E., Papo, M., Giaffer, M. H., SánchezLombraña, J. L., et al. (2002). Fat composition may be a clue to explain the primary therapeutic effect of enteral nutrition in Crohn's disease: results of a double blind randomised multicentre European trial. Gut 51, 164-168.

Gevers, D., Kugathasan, S., Denson, L. A., Vázquez-Baeza, Y., Van Treuren, W., Ren, B., et al. (2014). The treatment-naive microbiome in new-onset Crohn's disease. Cell Host Microbe 15, 382-392. doi: 10.1016/j.chom.2014.02.005

Harris, J. K., El Kasmi, K. C., Anderson, A. L., Devereaux, M. W., Fillon, S. A., Robertson, C. E., et al. (2014). Specific microbiome changes in a mouse model of parenteral nutrition associated liver injury and intestinal inflammation. PLoS ONE 9:e110396. doi: 10.1371/journal.pone.0110396

Hurt, R. T., and Wilson, T. (2012). Geriatric obesity: evaluating the evidence for the use of flavonoids to promote weight loss. J. Nutr. Gerontol. Geriatr. 31, 269-289. doi: 10.1080/21551197.2012.698222

Kaakoush, N. O., Day, A. S., Leach, S. T., Lemberg, D. A., Nielsen, S., and Mitchell, H. M. (2015). Effect of exclusive enteral nutrition on the microbiota of children 
with newly diagnosed Crohn's disease. Clin. Transl. Gastroenterol. 6, e71. doi: 10.1038/ctg.2014.21

Kwok, A. H., Li, Y., Jiang, J., Jiang, P., and Leung, F. C. (2014). Complete genome assembly and characterization of an outbreak strain of the causative agent of swine erysipelas-Erysipelothrix rhusiopathiae SY1027. BMC Microbiol. 14:176. doi: 10.1186/1471-2180-14-176

Labbé, A., Ganopolsky, J. G., Martoni, C. J., Prakash, S., and Jones, M. L. (2014). Bacterial bile metabolising gene abundance in Crohn's, ulcerative colitis and type 2 diabetes metagenomes. PLoS ONE 9:e115175. doi: 10.1371/journal.pone.0115175

Li, Y., Zhu, W., Gong, J., Zuo, L., Zhang, W., Gu, L., et al. (2014). Influence of exclusive enteral nutrition therapy on visceral fat in patients with Crohn's disease. Inflamm. Bowel Dis. 20, 1568-1574. doi: 10.1097/MIB.0000000000000114

Martínez, I., Perdicaro, D. J., Brown, A. W., Hammons, S., Carden, T. J., Carr, T. P., et al. (2013). Diet-induced alterations of host cholesterol metabolism are likely to affect the gut microbiota composition in hamsters. Appl. Environ. Microbiol. 79, 516-524. doi: 10.1128/AEM.03046-12

Martínez, I., Wallace, G., Zhang, C., Legge, R., Benson, A. K., Carr, T. P., et al. (2009). Diet-induced metabolic improvements in a hamster model of hypercholesterolemia are strongly linked to alterations of the gut microbiota. Appl. Environ. Microbiol. 75, 4175-4184. doi: 10.1128/AEM. 00380-09

Nahidi, L., Leach, S. T., Mitchell, H. M., Kaakoush, N. O., Lemberg, D. A., Munday, J. S., et al. (2013). Inflammatory bowel disease therapies and gut function in a colitis mouse model. Biomed. Res. Int. 2013:909613. doi: 10.1155/2013/ 909613

Nguyen, T. L., Vieira-Silva, S., Liston, A., and Raes, J. (2015). How informative is the mouse for human gut microbiota research? Dis. Model Mech. 8, 1-16. doi: $10.1242 / \mathrm{dmm} .017400$

Palm, N. W., de Zoete, M. R., Cullen, T. W., Barry, N. A., Stefanowski, J., Hao, L., et al. (2014). Immunoglobulin A coating identifies colitogenic bacteria in inflammatory bowel disease. Cell 158, 1000-1010. doi: 10.1016/j.cell.2014.08.006

Parmentier-Decrucq, E., Duhamel, A., Ernst, O., Fermont, C., Louvet, A., VernierMassouille, G., et al. (2009). Effects of infliximab therapy on abdominal fat and metabolic profile in patients with Crohn's disease. Inflamm. Bowel Dis. 15, 1476-1484. doi: 10.1002/ibd.20931
Schaubeck, M., Clavel, T., Calasan, J., Lagkouvardos, I., Haange, S. B., Jehmlich, N., et al. (2015). Dysbiotic gut microbiota causes transmissible Crohn's disease-like ileitis independent of failure in antimicrobial defence. Gut. doi: 10.1136/gutjnl2015-309333. [Epub ahead of print].

Spencer, M. D., Hamp, T. J., Reid, R. W., Fischer, L. M., Zeisel, S. H., and Fodor, A. A. (2011). Association between composition of the human gastrointestinal microbiome and development of fatty liver with choline deficiency. Gastroenterology 140, 976-986. doi: 10.1053/j.gastro.2010.11.049

Turnbaugh, P. J., Bäckhed, F., Fulton, L., and Gordon, J. I. (2008). Diet-induced obesity is linked to marked but reversible alterations in the mouse distal gut microbiome. Cell Host Microbe 3, 213-223. doi: 10.1016/j.chom.2008.02.015

Zhang, C., Zhang, M., Wang, S., Han, R., Cao, Y., Hua, W., et al. (2010). Interactions between gut microbiota, host genetics and diet relevant to development of metabolic syndromes in mice. ISME J. 4, 232-241. doi: 10.1038/ismej.2009.112

Zhang, H., DiBaise, J. K., Zuccolo, A., Kudrna, D., Braidotti, M., Yu, Y., et al. (2009). Human gut microbiota in obesity and after gastric bypass. Proc. Natl. Acad. Sci. U.S.A. 106, 2365-2370. doi: 10.1073/pnas.0812600106

Zhao, Y., Wu, J., Li, J. V., Zhou, N. Y., Tang, H., and Wang, Y. (2013). Gut microbiota composition modifies fecal metabolic profiles in mice. J. Proteome Res. 12, 2987-2999. doi: 10.1021/pr400263n

Zhu, Q., Jin, Z., Wu, W., Gao, R., Guo, B., Gao, Z., et al. (2014). Analysis of the intestinal lumen microbiota in an animal model of colorectal cancer. PLOS ONE 9:e90849. doi: 10.1371/journal.pone.0090849

Zschaler, J., Schlorke, D., and Arnhold, J. (2014). Differences in innate immune response between man and mouse. Crit. Rev. Immunol. 34, 433-454. doi: $10.1615 /$ critrevimmunol.2014011600

Conflict of Interest Statement: The author declares that the research was conducted in the absence of any commercial or financial relationships that could be construed as a potential conflict of interest.

Copyright (C) 2015 Kaakoush. This is an open-access article distributed under the terms of the Creative Commons Attribution License (CC BY). The use, distribution or reproduction in other forums is permitted, provided the original author(s) or licensor are credited and that the original publication in this journal is cited, in accordance with accepted academic practice. No use, distribution or reproduction is permitted which does not comply with these terms. 